transcarbamylase in liver of patients with Reye's syndrome. Pediatr. Res., 9 : 829 (1975).

30. Snodgrass, P. J. and DeLong, G.: Urea cycle deficiencies and an increased nitrogen load producing hyperammonemia in Reye's syndrome. N. Engl. J. Med., 294: 855 (1976).

31. Thaler, M. M. Personal communication.

32. Thaler, M. M.: Clinical and enzymatic indices of hepatic dysfunction in Reye's syndrome. In: John F. S. Crocker (Ed), Reye's syndrome II. pp. 115-137 (Grune \& Stratton, Inc., 1979).

33. Thaler, M. M., Hoogenraad, N. J., and Boswell, M.: Reye's syndrome due to a novel protein tolerant variant of ornithine-transcarbamylase deficiency. Lancet, 2: 438 (1974).
34. Requests for reprints should be addressed to: Dr. Donald M. Mock, Division of Gastroenterology and Nutrition, Department of Pediatrics, The University of Texas Health Science Center at San Antonio, 7703 Floyd Curl Drive, San Antonio, Texas 78284.

35. Supported by the National Institutes of Health Grant \# HDO 3148 and grant RR-00079 from the Division of Research Resources, National Institutes of Health, General Clinical Research Center. During a portion of Mock was the Clinical Associate Physician of the General Clinical Research Center.

36. Received for publication September 13, 1982.

37. Accepted for publication March 18, 1983.

0031-3998/83/1711-0888\$02.00/0

PEDIATRIC RESEARCH

Copyright (C) 1983 International Pediatric Research Foundation, Inc

Vol. 17, No. 11, 1983 Printed in U.S.A

\title{
Transcutaneous Bilirubinometry II. Dermal Bilirubin Kinetics during Phototherapy
}

\author{
THOMAS HEGYI, ${ }^{(20)}$ I. MARK HIATT, IAN M. GERTNER, ROBERT ZANNI, AND \\ THEORODORE TOLENTINO \\ Division of Neonatology, Department of Pediatrics, UMDNJ-Rutgers Medical School, and the Divisions of \\ Neonatology, Monmouth Medical Center and St. Peter's Medicial Center, New Brunswick, New Jersey, USA
}

\begin{abstract}
Summary
We examined the effect of phototherapy on cutaneous bilirubin using the transcutaneous bilirubinometer (TcB) in 14 white infants at a mean postnatal age of $30 \mathrm{~h}$. Six infants were treated with blue light, eight with white light. An opaque patch, $2.5 \mathrm{~cm}$ in diameter, covered the skin and served as a source for $T B$ control values. Simultaneous TcB measurements were obtained from exposed and patched areas every $15 \mathrm{~min}$ during $4 \mathrm{~h}$ of phototherapy.

After the start of phototherapy, TcB index obtained from patched skin did not change during the course of treatment. Data from exposed skin showed that the initial rate of TcB index fall during the first hour was significantly faster than the successive values during the next $3 \mathrm{~h}$ in both groups studied. During the first $2 \mathrm{~h}$ of therapy the TCB index decreased faster among infants treated with blue light.

Correlation studies indicate that TcB measurements from exposed skin areas may not be clinically useful in predicting serum bilirubin response to phototherapy but data obtained from unexposed sites may fill this role.
\end{abstract}

Previous studies have shown that visible light significantly reduces serum bilirubin concentrations in infants $(7,10)$. The photochemical reactions responsible for this effect, photodegradation and photoisomerization, occur primarily in exposed skin $(6,12)$. Shaded sites remain icteric and exposed areas are bleached under phototherapy (1).

In the present work the TCB (19) was used to monitor the responses of cutaneous bilirubin to phototherapy. The experiments were undertaken to determine the rate and the extent of skin decolorization and through direct skin measurements to help explain the model of action of phototherapy. This protocol was approved by the Institutional Committee on Research.

\section{MATERIALS AND METHODS}

Fourteen infants were studied. Birthweight ranged from 660 $3200 \mathrm{~g}$, gestation age from $26-41 \mathrm{wk}$, and postnatal age from 4 $63 \mathrm{~h}$. Patients were selected for study if they met the following criteria: (1) requirement for phototherapy to treat hyperbilirubinemia; (2) ability to maintain infant in a supine position for 4 $\mathrm{h}$; (3) availability of personnel to attend patient for $4 \mathrm{~h}$; and (4) ability to obtain consent form from mother or legal guardian. Infants with hemolytic anemia, positive Coombs test, or severe respiratory problems were excluded.

The infants were divided into two groups based on the type of phototherapy used. Group I was treated with blue light and Group II with white light. There were six infants in Group I, all nursed naked in incubators (Isolette, Air Shields, Hatboro PA). Eight special blue fluorescent lamps (Westinghouse, F-20T 12/ $\mathrm{BB}$ ) were encased in a standard nursery phototherapy module and positioned above the incubators. Group II consisted of eight infants nursed naked on radiant warmer beds (Infant Care Systems, Air Shields Co.) and treated with fluorescent bulbs housed in the phototherapy attachment (Air Shields, 78-800-70) of the warmer bed. Irradiance at the infant's level was measured with a photometer (Air Shields, Model PR-111). Radiant flux in the $425-475 \mathrm{~nm}$ band was $11.6 \pm 1.5 \mu \mathrm{W} \cdot \mathrm{cm}^{-2} \cdot \mathrm{nm}^{-1}$ (mean \pm S.D.) in Group I and $5.2 \pm 0.8 \mu \mathrm{W} \cdot \mathrm{cm}^{-2} \cdot \mathrm{nm}^{-1}$ in Group II. The difference between irradiances was significant at the $P<0.001$ level.

$\mathrm{TcB}$ index was measured on the exposed sternal area every 15 min during $4 \mathrm{~h}$ of phototherapy. Simultaneous measurements were obtained from a nearby site covered by an opaque patch $2.5 \mathrm{~cm}$ in diameter. The TcB's (Minolta, Japan) operating principles have been described previously (19). This spectral reflectometer measures the color intensity of the skin and provides a numerical $\mathrm{TcB}$ index that has been correlated to serum bilirubin concentrations $(5,19)$. 
Total serum bilirubin concentrations were measured once or twice during the course of phototherapy by a spectrophotometric technique corrected for hemolysis and for elevated concentrations of direct bilirubin (17). A total of 27 correlations were obtained, 10 in Group I and 17 in Group II. Of these 27, 17 were examined during the first hour of phototherapy, and 10 during the second through fourth hours.

We divided the duration of phototherapy into four hourly time periods and identified the rate of $\mathrm{TcB}$ index decline during these individual intervals by a linear regression between $T c B$ index and time. These periods were arbitrarily named A, B, C, and D and the respective $\mathrm{TcB}$ changes Slope A, Slope B, Slope C, and Slope D.

Results were analyzed with the Student $t$ test to compare mean

Table . Clinical data

\begin{tabular}{lcc}
\hline & Group I & Group II \\
\hline$n$ & 6 & 8 \\
Birthweight (g) (mean \pm S.D.) & $2280 \pm 710$ & $1740 \pm 610$ \\
Gestational age (wk) & $35 \pm 3$ & $32 \pm 3$ \\
Age at start of phototherapy (h) & $32.2 \pm 18.7$ & $29.3 \pm 16.6$ \\
SBC at start $(\mathrm{mg} / \mathrm{dl})$ & $8.0 \pm 2.5$ & $7.0 \pm 2.3$ \\
TCB initial & & \\
$\quad$ Patched & $18.5 \pm 4.4$ & $20.1 \pm 5.1$ \\
$\quad$ Unpatched & $18.5 \pm 4.9$ & $19.4 \pm 5.0$ \\
Light & Blue & White \\
Light intensity $\left(\mu \mathrm{W} \cdot \mathrm{cm}^{-2} \cdot \mathrm{nm}^{-1}\right)$ & $11.6 \pm 0.3$ & $5.2 \pm 0.8$ \\
\hline
\end{tabular}

${ }^{1}$ Abbreviations: $\mathrm{SBC}$, serum bilirubin concentration and $\mathrm{TcB}$, transcutaneous bilirubinometer. values and analysis of variance to examine the influence of different variables on Slope A. Post hoc test was performed by Duncan's multiple range test to compare the four slopes. The Pearson coefficient of correlation was used to relate $\mathrm{TcB}$ index and serum bilirubin concentrations.

\section{RESULTS}

In Table 1 we present the clinical characteristics of the patients studied. Infants exposed to blue light (Group I) were heavier and more mature than those treated with white light (Group II), but these differences did not reach statistically significant levels. The age at start of phototherapy, initial $\mathrm{TcB}$ indices, and initial serum bilirubin concentrations were comparable between the groups.

The rate of reduction in the $T c B$ indices in response to the two modes of phototherapy showed a similar pattern. After the start of therapy the $\mathrm{TcB}$ index obtained from the unexposed sites in both groups remained stable. The index from exposed areas decreased at a significantly faster rate during the first hour than during each of the next $3 \mathrm{~h}$. In Group I the initial rate of decline was $-3.4 \mathrm{TcB} \mathrm{U} / \mathrm{h}$ (Slope $\mathrm{A}$ ) and the fall during the second hour was $-1.6 \mathrm{TcB} U / \mathrm{h}$ (Slope B). During the third and fourth hours, $\mathrm{TcB}$ no longer decreased but remained stable $(+0.3$ and +0.4 $\mathrm{TcB} U / h$, respectively). In Group II, slope $A$ was $-2.7 \mathrm{TcB} U / \mathrm{h}$, slope $\mathrm{B}-0.5 \mathrm{TcB} \mathrm{U} / \mathrm{h}$, slope $\mathrm{C}-0.2 \mathrm{TcB} \mathrm{U} / \mathrm{h}$, and slope $\mathrm{D}-0.1$ $\mathrm{TcB} U / \mathrm{h}$. During the first $2 \mathrm{~h}$ the rate of TcB decline was faster in Group I infants. Figure 1 shows the mean TcB values of the two groups during the course of therapy. The response of serum bilirubin concentrations did not reflect the rapid decrease in $\mathrm{TcB}$. Evaluated after $90-140$ min of phototherapy, the SBC was $8.9 \pm$ $1.7 \mathrm{mg} / \mathrm{dl}$ in Group I and $8.0 \pm 1.9 \mathrm{mg} / \mathrm{dl}$ in Group II.

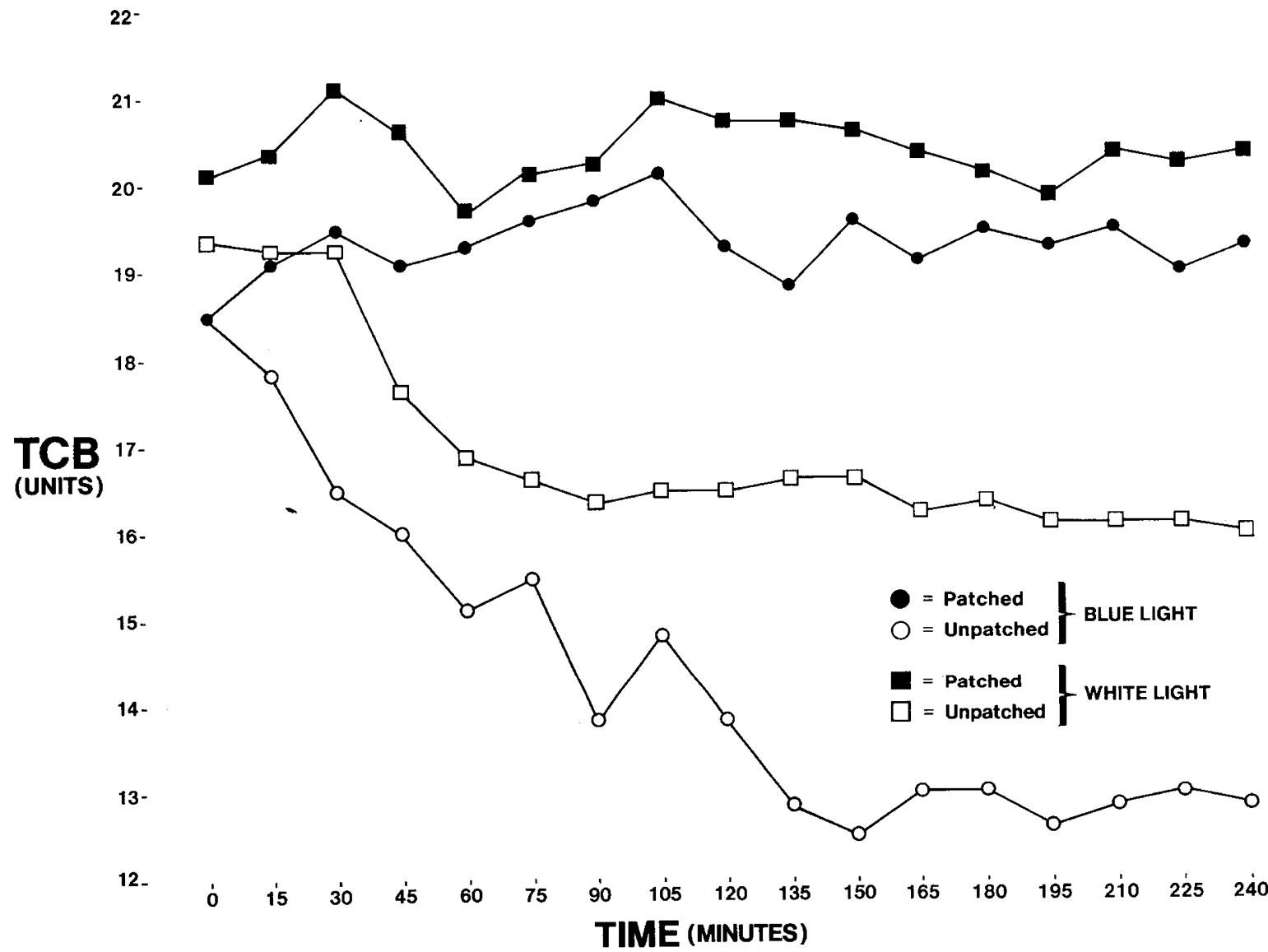

Fig. 1. The effect of two modes of phototherapy on transcutaneous bilirubinometer ( $T \mathrm{cB}$ ) indices. The zero point indicates the $T c B$ Index before start of phototherapy. Closed and open circles represent the mean values from patched and unpatched reas, respectively in Group I. Closed and open boxes represent mean values from patched and unpatched areas in Group II. 
The results of the study were analyzed to demonstrate the effect of the variables on Slope A. We found that in Group II Slope A correlated significantly with light intensity $(P<0.001)$. We could not demonstrate this relationship in the blue light group. Initial TcB index, birthweight, gestational age, and age at start of treatment did not significantly affect the results.

The correlation coefficients between the TcB indices and SBC were noted to be statistically significant. These coefficients in Group I were 0.90 and 0.71 comparing patched $T c B$ and unpatched $\mathrm{TcB}$, respectively, with SBC. In Group II these values were 0.68 and 0.52 .

\section{DISCUSSION}

The results of the study indicate that the rate of decrease of dermal bilirubin as measured by transcutaneous bilirubinometry is nonlinear with respect to duration of phototherapy. Serum bilirubin decreases in a similar pattern after the onset of phototherapy, an exponential decline that is dependent on the logarithm of the light dose (18). Skin bilirubin concentration decreases more rapidly than the plasma level, increasing the bilirubin gradient between skin and blood (14).

The stability of the TcB index of the unexposed area may imply that covered skin is not affected by phototherapy and that there is no lateral movement of bilirubin between two skin areas. Vogl (16), using the Gosset icterometer in his patching study found that light does not bleach the skin under a patch and a clear demarcation exists between bleached and icteric skin, suggesting that there is minimal lateral diffusion of bilirubin. Whereas the $\mathrm{TcB}$ from unexposed sites remained stable, we found the response from the exposed areas to be immediate. The bilirubin decrease in the unexposed areas followed the TcB index decline in the light treated areas by several hours. These findings with the TcB appear to agree with other indirect observations that bilirubin migration takes a number of hours $(3,16)$.

A closer analysis of the results of the study questions the above hypotheses. The premature infants were examined at an appropriate mean age of $30 \mathrm{~h}$ at a time when bilirubin concentration is expected to continually increase (2). The second evaluation of serum bilirubin concentration in this study showed higher levels compared to starting value. This rise in serum bilirubin concentration should be reflected in a continuing increase in the $T c B$ index or skin bilirubin of the patched areas, especially because the relatively low starting serum bilirubin concentrations would not saturate all skin binding sites. Although this increase was not observed, the abrupt halt in $\mathrm{TcB}$ rise suggests that bilirubin diffuses into the blood stream more rapidly than expected or the lateral diffusion of bilirubin between skin sites has been underestimated. The clear line of demarcation between the two sites, as noted by Vogl (16), may be caused by the instantaneous isomerization of the pigmented compound on exposure to light (8).

The proposed mechanism for phototherapy by McDonagh and colleagues (13) helps to explain some of the study findings. Photochemical excitation of bilirubin yields an unstable compound that decays to ground state bilirubin or, after isomerization, to ground state photobilirubin. This isomerized compound then shifts into the circulation, a phenomenon observed clinically as bleaching. This movement appears to be a diffusion controlled process, bi-directional and rapid. The departed photobilirubin is replaced in the skin by bilirubin from the blood. Photobilirubin is then excreted by the liver and can revert back to the natural compound in the bile or in the gastrointestinal tract.

The response to phototherapy depends on wavelengths, light intensity, surface area exposed, and rates at which the isomerized bilirubin molecule is removed from skin and blood. After exposure to light, both groups achieve a steady state as measured by the TcB. Here, the rates of pigment deposition and removal are equal. The rate of skin bleaching is dependent on the rate of photoisomer formation which in turn is affected by light inten- sity. Increasing light intensity increases bilirubin isomerization resulting in a different steady state level. The faster rate of TcB decline and the longer duration of effectiveness in the blue light group confirm the importance of radiant flux in determining the response (15).

Bilirubin is not distinguished from its photoisomers by conventional laboratory methods (12). Total bilirubin concentrations represent a combination of the toxic natural compound and the less toxic photoisomer. Therefore, the changes in total serum bilirubin concentrations do not reflect the efficiency of phototherapy. The changes in the indices therefore show a changing kinetic equilibrium; however, it is possible that altered photochemical equilibrium is also reflected: the decreasing concentration of natural bilirubin and the increasing concentration of the photoisomer. The total skin concentration of these compounds is limited by the presence of a finite number of available binding sites (9). If the reaction is limited by the transport of photobilirubin either from skin to blood or from blood to bile, then the exposed area will have a constant isomerized compound population. This stable relationship between the different isomers may explain the stable TcB index of the exposed areas reached after $90 \mathrm{~min}$ in the white light group and $135 \mathrm{~min}$ in the blue light group. The increased effectiveness of higher intensity may be due to increased skin penetrance and effect on more sites.

In view of the present findings, the $T c B$ acts as a different monitor for phototherapy because changes in total serum bilirubin concentration measurements may not be accurately reflected but rate of skin bleaching can be monitored.

Attempts at correlating the $\mathrm{TcB}$ index to serum bilirubin concentration on and even off phototherapy have yielded inconsistent results. Several recent reports have provided statistically significant correlations, with coefficients of correlation ranging from $0.72-0.95(4,5,11,19)$. A surprising result of Hanneman and coworkers' study (4) was a correlation of 0.86 of TcB index and serum bilirubin concentrations in infants treated with phototherapy. In an earlier report we questioned the value of $\mathrm{T} C B$ measurements under phototherapy because our correlation studies did not reach statistically significant levels (5). In the present work, in spite of the heterogeneous characteristics and limited numbers of the infants studied, we achieved statistically significant $(P<0.05)$ correlation between TcB measurements from exposed skin and serum bilirubin levels. We still believe this use of the TcB to predict serum bilirubin concentration to be of dubious value. Correlations obtained from unexposed skin may fill this need but further studies are necessary.

\section{REFERENCES AND NOTES}

1. Bajpai, P. C., Agarwal, S. S., Kapoor, C. L., and Krishna Murti. C. R.: Skin as the site of photoconversion of bilirubin in hyperbilirubinemia of the newborn. India J. Med. Res., 64: 1214 (1976).

2. Brown, A. K.: Bilirubin metabolism in the fetus and newborn in Smith, C. A. and Nelson, N. M. eds: The Physiology of the Newborn Infant. p. 315 (Charles C Thomas, Springfield, 1976).

3. Brown, A. K., Zuelzer, W. W., and Robinson, A. R.: Studies in hyperbilirubinemia 11. Clearance of bilirubin from plasma and extravascular space in newborn. J. Dis. Child., 93: 274 (1957).

4. Hanneman, R. E., Schreiner, R. L., Dewitt, D. P., Norris, S. A., and Glick, M. R.: Evaluation of the Minolta bilirubin meter as a screening device in white and black infants. Pediatrics, 89: 107 (1982).

5. Hegyi, T., Hiatt, I. M., and Indyk, L.: Transcutaneous bilirubinometry 1. Correlations in term infants. J. Pediatr., 98: 454 (1981).

6. Hewitt, J. R., Klein, R. M., and Lucey, J. F.: Photodegradation of serum bilirubin in the Gunn rat. Biol. Neonate, 21: 112 (1972).

7. Hodgman, J. E. and Schwartz, A.: Phototherapy and hyperbilirubinemia of the premature. Am. J. Dis. Child., 119:473 (1970).

8. Indyk, L.: Physical aspects of phototherapy. In Bergsma D. and Blondstein, S. H.. (eds) Bilirubin metabolism in the newbron (11) p. 23 (Amsterdam, 1976, The National Foundation/March of Dimes, Excerpta Medica).

9. Kapoor, C. L., Murti, C. R. K., and Bajpai, P. C.: Uptake and release of bilirubin by skin. Biochem. J., 136: 35 (1973).

10. Lucey, J. F., Ferreiro, M., and Hewitt, J.: Prevention of hyperbilirubinemia of prematurity by phototherapy. Pediatrics, 41: 1047 (1980).

11. Lucey, J. F.. Nyborg, E., and Yamonouchi, I.: A new device for transcutaneous bilirubinometry. Pediatr. Res., 14: 604 (1980). 
12. McDonagh; A. F.: Phototherapy: a new twist to bilirubin. J. Pediatr.. 99: 909 (1981).

13. McDonagh A. F. Palma, L. A., and Lightner, D. A.: Blue light and bilirubin excretion. Science, 208: 145 (1980).

14. Rubaltelli, F. F. and Carli, M.: The effect of light on cutaneous bilirubin. Biol. Neonate, 18: 457 (1971).

15. Sisson, T. R. C., Kendall, N., Shaw, E., and Kechavarz-Oliai, L.: Phototherapy of jaundice in the newborn infant: 11 Effect of various light intensities. J. Pediatr., 81: 35 (1972).

16. Vogl, T. P.: Phototherapy of neonatal hyperbilirubinemia. Bilirubin in unexposed areas of the skin. J. Pediatr., 85: 707 (1974).

17. White D.Hardar G. A., and Reinhold, J. G.: Spectrophotometric measure- ments of bilirubin concentrations in the serum of the newborn by the use of a microcapillary method. Clin. Chem., 4: 211 (1958).

18. Wiese, G. and Ballowitz, L.: Phototherapy in Gunn rats 11 . Further calculations on the effectivity of different irradiances. Biol. Neonate, 39: 113 (1981).

19. Yamanouchi, I. Yamauchi, Y., and Igarashi, I.: Transcutaneous bilirubinometry: Preliminary studies of noninvasive transcutaneous bilirubin meter in Okayama National Hospital. Pediatrics, 65: 195 (1980).

20. Requests for reprints should be addressed to: Dr. Thomas Hegyi, Director, Division of Neonatal Medicine, UMDNJ-Rutgers Medical School, St. Peter's Medical Center, 254 Eastern Ave., New Brunswick, NJ 08903.

21. Received for publication October 6, 1982.

22. Accepted for publication April 6, 1983

\title{
Energy Balance and Nitrogen Balance in Growing Low Birthweight Infants Fed Human Milk or Formula
}

\author{
R. K. WHYTE, ${ }^{(40.41)}$ R. HASLAM, C. VLAINIC, S. SHANNON, K. SAMULSKI, D. CAMPBELL, \\ H. S. BAYLEY, AND J. C. SINCLAIR \\ Department of Pediatrics, McMaster University, Hamilton, Ontario and Department of Nutrition, University of \\ Guelph, Guelph, Ontario, Canada
}

\begin{abstract}
Summary
Energy and nitrogen balances were measured in growing low birthweight infants fed either mother's expressed breast milk or a $20 \mathrm{kcal}$ per ounce formula to determine whether or not there were differences between the two dietary groups in (1) the partition of energy among excretion, expenditure, and storage and (2) the relation of energy storage and nitrogen retention to weight gain.

There were no significant differences between the human milk fed infants and formula fed infants in gross energy intake, metabolizable energy intake, nitrogen intake, or nitrogen retention. Energy expenditure was significantly lower in the human milk fed infants than in formula fed infants $(221 \mathrm{~kJ} /(\mathrm{kg}$. day $)$ and 244 $\mathrm{kJ} /(\mathrm{kg}$. day), respectively). There was no difference in mean energy storage between the two groups.

Although weight gains were similar in both dietary groups, the ratio of energy storage to weight gain was significantly greater in infants fed with human milk $(15.3 \mathrm{~kJ} / \mathrm{g}$, S.D. 2.0) than in infants fed formula (13.2 kJ/g S.D. 1.8). There was no significant difference between the two groups in the ratio of nitrogen stored to weight gain.
\end{abstract}

Estimates of the body composition of the growing fetus (38) have been used as guidelines from which the nutrient requirements of the growing low birthweight infant have been calculated (15). This approach has been challenged (21): the low birthweight infant in an extrauterine environment, with physiologic and environmental conditions differing from those of the fetus, may have different nutrient requirements both for maintenance and for storage in growing tissues. The observation that human milk expressed from preterm mothers differs from that of term mothers $(1,2,3,17)$ has contributed to a renewed interest in the suitability of this food for growing low birthweight infants. Pre- vious studies $(8,28)$ have measured energy balance in growing low birthweight infants fed formula. In this study we have measured both energy and nitrogen balances in growing low birthweight infants fed either their own mother's expressed milk or formula.

The aims of the study are to compare the two dietary groups with regard to $(l)$ the partition of energy intake into energy excretion, expenditure, and storage; $(2)$ the partition of nitrogen intake into excretion and retention; and (3) the relation of energy storage and nitrogen retention to weight gain. From these measurements, the composition of weight gain was estimated for each dietary group.

\section{SUBJECTS AND METHODS}

Informed consent was sought from the parents of healthy, growing, low birthweight infants born at or referred to McMaster University Medical Centre between June 1978 and August 1980. To qualify for the study, each baby had to be receiving all feeds by gavage, free of respiratory or gastrointestinal disease, less than $1800 \mathrm{~g}$ at birth and growing at a rate of at least $10 \mathrm{~g} /(\mathrm{kg}$. day).

Allocation was by maternal preference into a human milk fed group and a formula fed group. Formula (Similac, Ross Laboratories) was obtained after reconstitition and resterilization from a distribution service. Only those infants who were fed exclusively either with formula or with their own mother's expressed milk were included in the study.

Each study consisted of a 14-day balance period during which time the amount of feed intake was measured daily (Fig. 1). Pooled samples of feed and of excreta were collected for analysis during collection periods which comprised the first 3 and the last 3 days of the balance period. Energy expenditure was measured three times, at weekly intervals, on each occasion throughout two interfeed periods ( 4 or $6 \mathrm{~h}$ ). Behavioural state, observed 